\title{
Isomorphic random subspaces and quotients of convex and quasi-convex bodies.
}

\author{
Alexander E. Litvak Vitali D. Milman \\ Nicole Tomczak-Jaegermann*
}

\begin{abstract}
We extend the results of [LMT] to the non-symmetric and quasiconvex cases. Namely, we consider finite-dimensional space endowed with gauge of either closed convex body (not necessarily symmetric) or closed symmetric quasi-convex body. We show that if a generic subspace of some fixed proportional dimension of one such space is isomorphic to a generic quotient of some proportional dimension of another space then for any proportion arbitrarily close to 1, the first space has a lot of Euclidean subspaces and the second space has a lot of Euclidean quotients.
\end{abstract}

\section{Introduction}

The present paper is a continuation of the study that began in [LMT], and which dealt with phenomena suggested by Bourgain and Milman in [BM1]. Recall that they proved that given any two normed spaces $X$ and $Y$, for a large set of (proportional dimensional) subspaces of $X$ and a large set of quotients of $Y$, the distance between any two representatives is much less than can be expected in the general case by Gluskin's theorem ([Gl]). In particular, it showed that "random" subspaces and "random" quotients are not of the same nature, and should have some very different properties.

In [LMT] we answered a vaguely posed question from [BM1]. In the present paper we extend the results of [LMT] to the non-symmetric and

*This author holds The Canada Research Chair in Geometric Analysis. 
quasi-convex cases. More precisely, we show that if, for some random structure (described below), a generic subspace of some fixed proportional dimension is isomorphic (essentially the same) to a generic quotient of some proportional dimension of another space (with a similarly selected random structure) then for any proportion arbitrarily close to 1, the first space has a lot of Euclidean subspaces and the second space has a lot of Euclidean quotients. So a complete similarity between a generic subspace and a generic quotient implies that most subspaces (respectively, quotients) are Euclidean.

The organization of the paper is as follows. In Sections 1 and 2 we recall some basic notations and some "symmetric" auxiliary results from [LMT]. Next we discuss the non-symmetric case in Sections 3 and 4. For completeness and future references we provide all proofs. Our main theorem in the nonsymmetric case is Theorem 4.1. Then in Section 5 we discuss some possible technical improvements of results from [LMT] and dependence of constants on parameters. Finally, in Section 6, we show that our results hold in the symmetric quasi-convex case as well.

The asymptotic theory of non-symmetric convex bodies was developed in [MPa1, MPa2], [LT], [Ru]. We refer to these papers and references therein for all background not explained here. For quasi-convex bodies many results of the asymptotic theory extend as well ([BBP], [D], [GK], [KPR], [M6]); we shall give more specific references futher in the text.

Acknowledgement The authors would like to thank to E. D. Gluskin for useful dscussions and critical remarks on the first version of the paper.

\section{Definitions and notations}

We consider $\mathbb{R}^{n}$ with the standard Euclidean structure and the Euclidean unit ball denoted by $B_{2}$. The canonical Euclidean norm on $\mathbb{R}^{n}$ is denoted by $|\cdot|$, and the corresponding inner product by $\langle\cdot, \cdot\rangle$. We shall also consider other Euclidean structures on $\mathbb{R}^{n}$, with the unit balls given by ellipsoids.

In this paper by a body we always mean a compact star-shaped set with 0 in its interior. This means that from the very beginning we choose and fix the center of the body in the origin and all definitions below will not allow shifts. We shall call a convex body symmetric if it is centrally symmetric. For a convex body $K$ in $\mathbb{R}^{n}$ the polar body $K^{0}$ is defined by

$$
K^{0}:=\left\{x \in \mathbb{R}^{n} \mid\langle x, y\rangle \leq 1 \text { for every } y \in K\right\} .
$$


Recall that for every subspace $E$ of $\mathbb{R}^{n}$ the polar (in $E$ ) of $K \cap E$ is $P_{E} K^{0}$, where $P_{E}$ is the orthogonal projection onto $E$.

The $n$-dimensional volume of a body $K$ in $\mathbb{R}^{n}$ is denoted by $|K|$. For a body $K \subset \mathbb{R}^{n}$ we shall occasionally use the notation $\|\cdot\|_{K}$ for the Minkowski functional of $K$. The space $\left(\mathbb{R}^{n},\|\cdot\|_{K}\right)$ will be also denoted by $\left(\mathbb{R}^{n}, K\right)$. If $L \subset \mathbb{R}^{m}$ is another body and $T: \mathbb{R}^{n} \rightarrow \mathbb{R}^{m}$ is a linear operator, by $\|T: K \rightarrow L\|$ we shall denote the operator norm of $T$ from $\left(\mathbb{R}^{n}, K\right)$ to $\left(\mathbb{R}^{m}, L\right)$. If $m=n$, the geometric distance between $K$ and $L$ is defined by

$$
d_{g}(K, L):=\inf \{b / a \mid a>0, b>0, a K \subset L \subset b K\} .
$$

If $d_{g}(K, L) \leq C$ then we say that $K$ and $L$ are $C$-equivalent. In this paper we define Banach-Mazur distance between $K$ and $L$ by

$$
d(K, L):=\inf \left\{d_{g}(K, T L)\right\}
$$

where the infimum is taken over all invertible linear operators $T$ from $\mathbb{R}^{n}$ to $\mathbb{R}^{n}$. Note again that, compared with the usual definition, we do not minimize over shifts in the infimum above. The Banach-Mazur distance between spaces is the Banach-Mazur distance between their unit balls. If the Banach-Mazur distance between a space and the Euclidean space is bounded by $C$ we say that the space is $C$-Euclidean.

For a real number $a>0$, by $\lceil a\rceil$ we denote the smallest integer larger than or equal to $a$.

In this paper we consider ellipsoids centered at 0 only. Given an ellipsoid $\mathcal{E}$ on $\mathbb{R}^{n}$, by $G_{n, k}^{\mathcal{E}}$ for $1 \leq k \leq n$ we shall denote the Grassman manifold of $k$-dimensional linear subspaces of $\mathbb{R}^{n}$ equiped with the normalized Haar measure $\mu_{n, k}^{\mathcal{E}}$ determined by the Euclidean structure given by $\mathcal{E}$. If $\mathcal{E}=B_{2}$ we shall write $G_{n, k}$ and $\mu_{n, k}$ instead of $G_{n, k}^{\mathcal{E}}$, and $\mu_{n, k}^{\mathcal{E}}$. We say that some property holds for a random orthogonal (in $\mathcal{E}$ ) projection of rank $k$ whenever the measure of the set of all subspaces $E \in G_{n, k}^{\mathcal{E}}$ for which $P_{E}$ has the property, is larger than $1-\exp (c k)$ for some absolute constant $c>0$.

For a body $K \subset \mathbb{R}^{n}$ (recall that $K$ contains origin as an interiour point), by $\mathcal{E}_{K} \supset K$ and $\mathcal{E}_{K}^{\prime} \subset K$ we denote ellipsoids of minimal and maximal volume for $K$ respectively. Note that we consider minimal and maximal volume ellipsoids centered at origin, i.e. we don't allow shifts. We would like also to note that for convex bodies such ellipsoids are uniquely defined. It implies that the minimal volume ellipsoid is unique for any compact starshaped body $K \subset \mathbb{R}^{n}$ (since it coinsides with the ellipsoid of minimal volume 
for the convex hull of the body). In general, in the non-convex case, maximal volume ellipsoid is not necessarily unique, so dealing with non-convex bodies we choose and fix one of them

The volume ratio of $K$ and the outer volume ratio of $K$, with respect to the origin, are defined by

$$
\operatorname{vr}(K):=\left(|K| /\left|\mathcal{E}_{K}^{\prime}\right|\right)^{1 / n} \quad \text { and } \quad \operatorname{outvr}(K):=\left(\left|\mathcal{E}_{K}\right| /|K|\right)^{1 / n} .
$$

For a body $K \subset \mathbb{R}^{n}$, an ellipsoid $\mathcal{E}$ on $\mathbb{R}^{n}$, and any $0<\lambda<1$ we shall consider certain subsets $\mathcal{F}_{\lceil\lambda n\rceil}(K) \subset G_{n,\lceil\lambda n\rceil}^{\mathcal{E}}$ of $\lceil\lambda n\rceil$-dimensional subspaces of $\mathbb{R}^{n}$. Each element of $\mathcal{F}_{\lceil\lambda n\rceil}(K)$ gives rise to two different normed spaces. Firstly, it can be treated as a subspace of the normed space $\left(\mathbb{R}^{n}, K\right)$, in which case we may use a generic notation $s K$, that is, $s K:=(E, K \cap E)$. The set of all these subspaces will be denoted by $\mathcal{F}_{s,\lceil\lambda n\rceil}(K)$. Secondly, every $E \in \mathcal{F}_{\lceil\lambda n\rceil}(K)$ gives rise to a quotient space of $\left(\mathbb{R}^{n}, K\right)$, via the orthogonal (in $\mathcal{E}$ ) projection $P_{E}$ onto $E$, and in this case we may use a generic notation of $q K$; that is, $q K:=\left(E, P_{E} K\right)$. The set of all these quotient spaces will be denoted by $\mathcal{F}_{q,\lceil\lambda n\rceil}(K)$. (It should be noted that given a family $\mathcal{F}_{k}$, the definition of $\mathcal{F}_{s, k}$ does not depend on the ellipsoid $\mathcal{E}$, while the definition of $\mathcal{F}_{q, k}$ depends on this ellipsoid in an essential way.)

\section{The minimal volume ellipsoid of a symmetric convex body}

In this section we briefly recall properties of the minimal (resp., maximal) volume ellipsoid associated to a symmetric convex body, which we proved in [LMT]. These properties will play an essential role in our constructions. They deal with relations to any other ellipsoid containing (resp., contained in) the same body. These new properties depend on an abstract condition of Dvoretzky-Rogers-type. All results can be dualized in a standard way to the corresponding statements for the maximal volume ellipsoids and their sections.

Let $B$ be a symmetric convex body in $\mathbb{R}^{m}$ and let $\mathcal{E} \subset \mathbb{R}^{m}$ be an ellipsoid. Let $\phi:(0,1] \rightarrow(0,1]$ be a function. We say that $\mathcal{E}$ has property $(*)$ with respect to $B$ with function $\phi$, whenever

(*) for any $1 \leq k \leq m$ and any projection $Q$ of rank $k$ on $\mathbb{R}^{m}$ orthogonal with respect to $\mathcal{E}$ we have $\|Q: B \rightarrow \mathcal{E}\| \geq \phi(k / m)$. 
It is well known that the minimal volume ellipsoid satisfies $(*)$ with the function $\phi(t)=\sqrt{t}$. This is connected to, but simpler than, the DvoretzkyRogers Lemma. (Lemma 2.2 below shows that proportional-dimensional projections of the minimal volume ellipsoids satisfy $(*)$ as well.)

Theorem 2.1 Let $\mathcal{E} \subset \mathbb{R}^{m}$ and $\mathcal{D} \subset \mathbb{R}^{m}$ be two ellipsoids, let $B:=\mathcal{E} \cap \mathcal{D}$. Let $\phi:(0,1] \rightarrow(0,1]$ and set $A:=\left(\prod_{l=1}^{m} \phi(l / m)\right)^{-1 / m}$. Assume that $\mathcal{E}$ has property $(*)$ with respect to $B$ with function $\phi$. Then

$$
|\mathcal{E}|^{1 / m} \leq A|\mathcal{D}|^{1 / m}
$$

A typical situation when this theorem may be used is when a symmetric convex body $\tilde{B} \subset \mathbb{R}^{m}$ is given, $\mathcal{E} \supset \tilde{B}$ is any ellipsoid satisfying property (*) with respect to $\tilde{B}$ (see e.g., Lemma 2.2 below), and $\mathcal{D} \supset \tilde{B}$ is arbitrary.

As already mentioned, the minimal volume ellipsoid satisfies $(*)$ with $\phi(t)=\sqrt{t}$. A more general class of examples is provided by proportionaldimensional projections of the minimal volume ellipsoids.

Lemma 2.2 Let $K \subset \mathbb{R}^{n}$ be a symmetric convex body and let $\mathcal{E}_{K}$ be the ellipsoid of minimal volume for $K$. Let $P$ be an arbitrary projection in $\mathbb{R}^{n}$ with rank $P=m=\alpha$, for some $0<\alpha \leq 1$. Then $P \mathcal{E}_{K}$ has property (*) with respect to $P K$ with function $\phi(t)=\sqrt{\alpha t}$.

For future reference we formulate an important case.

Corollary 2.3 Let $m \leq n=\beta m$ for some $\beta \geq 1$. Let $K \subset \mathbb{R}^{n}$ be a symmetric convex body and let $\mathcal{E}_{K}$ be the ellipsoid of minimal volume for $K$. Let $P$ be an arbitrary projection in $\mathbb{R}^{n}$ with rank $P=m$. Set $E=P\left(\mathbb{R}^{n}\right)$ and $\mathcal{E}=P\left(\mathcal{E}_{K}\right)$. Let $\mathcal{D} \subset E$ be an arbitrary ellipsoid such that $\mathcal{D} \supset P K$. Then

$$
|\mathcal{E}|^{1 / m} \leq c \sqrt{\beta}|\mathcal{D}|^{1 / m}
$$

where $c>0$ is an absolute constant.

\section{Convex bodies in $M$-position}

Let us first recall the definition and a few basic facts about $M$-ellipsoids and $M$-positions of convex (not necessarily symmetric) bodies. 
Let $K$ and $L$ be two sets on $\mathbb{R}^{n}$. By $N(K, L)$ we denote the covering number, i.e. the minimal number of translations of $L$ needed to cover $K$.

Let $K \subset \mathbb{R}^{n}$ be a convex body and let $C>0$. We say that $B_{2}$ is an $M$-ellipsoid for $K$ with constant $C$ if we have

$$
\max \left\{N\left(K, B_{2}\right), N\left(B_{2}, K\right), N\left(K^{0}, B_{2}\right), N\left(B_{2}, K^{0}\right)\right\} \leq \exp (C n) .
$$

In this case we shall often say that $K$ is in $M$-position with constant $C$. It is a deep theorem first proved in [M3] that there is an absolute constant $C_{0}>0$ such that for every symmetric convex body $K$ in $\mathbb{R}^{n}$ there exists a linear transformation taking $K$ into $M$-position with constant $C_{0}$. In the non-symmetric case the existence of $C_{0}>0$ such that for any convex body $K$ there exists an affine transformation taking $K$ into $M$-position was proved in $[\mathrm{MPa} 1, \mathrm{MPa} 2]$ and independently in $[\mathrm{Ru}]$. Moreover, the positions constructed in these papers determine a choice of the center at the barycenter or at a point close to the barycenter (say $0 \in(K+z) / 2$, where $z$ is the barycenter) and, assuming as we do in this paper that the center is the origin, (3.1) holds for $K-K$ and $K \cap(-K)$ as well. (This is a consequence of the fact that bodies $K \cap(-K), K$ and $K-K$ have the same volume (up to $c^{n}$ ), whenever the origin is the barycenter of $K$, or a point close to the barycenter.) So we may and shall assume, without loss of generality, that in $M$-position the center is an interior point of $K$ and is fixed at the origin, and $K, K-K$ and $K \cap(-K)$ satisfy (3.1).

Throughout the paper we shall use the notation $C_{0}$ for such a constant in (3.1). However we shall often omit to mention it explicitely and we may just write, for example, that $K$ is in $M$-position. Still, the reader should always remember that from now on all our absolute constants later actually depend on this $C_{0}$.

It follows from the definition that if $K$ is in $M$-position then so is $K^{0}$ and that

$$
\left|e^{-C_{0}} B_{2}\right| \leq \min \left(|K|,\left|K^{0}\right|\right) \leq \max \left(|K|,\left|K^{0}\right|\right) \leq\left|e^{C_{0}} B_{2}\right| \text {. }
$$

Without loss of generality we assume from now on that whenever $K$ is in $M$-position then $|K|=\left|B_{2}\right|$.

In fact the estimates (3.1) are consequences of the conditions $|K|=\left|B_{2}\right|$ and one estimate $N\left(K, B_{2}\right) \leq \exp \left(C_{0}^{\prime} n\right)$ with $C_{0}^{\prime}>0$ (see Lemma 4.2 of [MS2] for the symmetric case and Lemma 10 and Remark 1 that follows in [MPa2] for the general case). 
As in $[\mathrm{LMT}]$ we shall use that for any two sets in $\mathbb{R}^{n}$ and every projection $P$ and every subspace $E$ one has

$$
N(P K, P L) \leq N(K, L) \quad \text { and } \quad N(K \cap E,(L-L) \cap E) \leq N(K, L) .
$$

We now extend a basic functorial construction from [LMT] to the nonsymmetric case. For each symmetric convex body $K$ in $\mathbb{R}^{n}$ in $M$-position and every $0<\lambda<1$ we shall define a certain subset $\mathcal{F}_{\lceil\lambda n\rceil}(K) \subset G_{n,\lceil\lambda n\rceil}$ such that

$$
\mu_{n,\lceil\lambda n\rceil}\left(\mathcal{F}_{\lceil\lambda n\rceil}(K)\right) \geq 1-e^{-c_{\lambda} n},
$$

where $c_{\lambda}>0$ is a function of $\lambda$ only. In the future we shall refer to a subset satisfying measure estimates of this type as a random family.

Given $K \subset \mathbb{R}^{n}$ as above, recall that the ellipsoids of minimal and maximal volume for $K$ (with respect to the origin) are denoted by $\mathcal{E}_{K} \supset K$ and $\mathcal{E}_{K}^{\prime} \subset K$, respectively. We shall denote the semi-axes of $\mathcal{E}_{K}$ by $\rho_{1} \geq \rho_{2} \geq$ $\ldots \geq \rho_{n}$ and a corresponding orthonormal basis by $\left\{e_{i}\right\}_{i=1}^{n}$. Similar notation is adopted for $\mathcal{E}_{K}^{\prime}$ with the semi-axes $\rho_{1}^{\prime} \geq \rho_{2}^{\prime} \geq \ldots \geq \rho_{n}^{\prime}$ and a corresponding orthonormal basis $\left\{e_{i}^{\prime}\right\}_{i=1}^{n}$.

Define $\mathcal{F}_{\lceil\lambda n\rceil}(K)$ as the set of all $E \in G_{n,\lceil\lambda n\rceil}$ satisfying

(i) $P_{E} B_{2} \subset C_{\lambda} P_{E} K$, where $P_{E}$ is orthogonal in $B_{2}$;

(ii) $K \cap E \subset C_{\lambda} B_{2} \cap E$;

(iii) $\left|P_{E} x\right| \geq b_{\lambda}|x|$ for every $x \in \operatorname{span}\left\{e_{i}\right\}_{i=1}^{m} \cup \operatorname{span}\left\{e_{i}^{\prime}\right\}_{i=n-m+1}^{n}$, where $m=\lceil\lambda n / 2\rceil$,

where $C_{\lambda}>0$ is an appropriate function on $\lambda$, and $b_{\lambda}=c_{0} \sqrt{\lambda}$ with an appropriate absolute constant $c_{0}>0$. Below we keep the notation $C_{\lambda}, b_{\lambda}$ and $c_{0}$ for these constants.

Proposition 3.1 Let $K$ be a convex body in $M$-position. Then there exist a choice of $C_{\lambda}, c_{\lambda}$ and $c_{0}$ such that the corresponding family $\mathcal{F}_{\lceil\lambda n\rceil}(K)$ satisfies (3.3).

For the proof of this proposition in the symmetric case see [LMT]. It was shown in [MPa2] that the set of subspaces satisfying conditions (i) and (ii) has measure larger than $1-2 e^{-n}$. Since condition (iii) is the general fact about orthogonal projections and doesn't depend on the body (see [LMT]) 
we obtain Proposition 3.1 for the non-symmetric case as well. We would like to note here that estimates in [MPa1, MPa2] and [LMT] gives that $C_{\lambda}$ can be chosen such that $C_{\lambda} \leq c^{1 /(1-\lambda)}$, where $c$ is an absolute positive constant. We discuss in section 5 below how one can improve the dependence of $C_{\lambda}$ on $\lambda$ in the symmetric case.

\section{Main results in the non-symmetric case}

In this section we extend the main result of [LMT] to the non-symmetric case. The proofs of the results basically follow the same lines with minor modifications and we include all proofs for completeness and future references.

Theorem 4.1 Let $K$ and $L$ be two convex bodies in $\mathbb{R}^{n}$ in $M$-position, and assume that for some $0<\lambda<1$ and some $d>1$ there is a quotient space $q K \in \mathcal{F}_{q,\lceil\lambda n\rceil}(K)$ and a subspace $s L \in \mathcal{F}_{s,\lceil\lambda n\rceil}(L)$ such that the Banach-Mazur distance satisfies

$$
d(q K, s L) \leq d
$$

Then

$$
\operatorname{outvr}(K) \leq C \quad \text { and } \quad \operatorname{vr}(L) \leq C,
$$

where $C=C(\lambda, d)$ is a function of $\lambda$ and $d$ only.

The proof of the theorem is based on the following proposition.

Proposition 4.2 Let $K \subset \mathbb{R}^{n}$ be a convex body in $M$-position. Let $0<\lambda<$ 1. Let $E \in \mathcal{F}_{\lceil\lambda n\rceil}(K)$ and let $P_{E}$ be the orthogonal projection on $E$. Then

$$
\operatorname{outvr}(K) \leq C_{\lambda}^{\prime}\left(\operatorname{outvr}\left(P_{E} K\right)\right)^{2}
$$

where $C_{\lambda}^{\prime}$ depends on $\lambda$ (and on constant $C_{0}$ which defines the $M$-position we use).

In other words, the proposition says that if $K$ is in $M$-position then for any quotient space $q K \in \mathcal{F}_{q,\lceil\lambda n\rceil}(K)$ we have outvr $(K) \leq C_{\lambda}^{\prime}(\operatorname{outvr}(q K))^{2}$. Recall here that our outer volume ratio is in fact outer volume ratio with respect to the origin.

Remark As it can be seen from the proof below, the power 2 in the estimate (4.1) can be improved to any $\alpha>1$. 
Proof of Proposition 4.2 Recall that $\mathcal{E}_{K} \supset K$ is the ellipsoid of minimal volume for $K$, and we denoted its semi-axes by $\rho_{1} \geq \rho_{2} \geq \ldots \geq \rho_{n}$, and the corresponding orthonormal basis by $\left\{e_{i}\right\}_{i=1}^{n}$. To simplify the notation, set $P:=P_{E}$. Consider the ellipsoid $P \mathcal{E}_{K}$ in $E$, and denote its semi-axes by $\rho_{1}^{\prime} \geq \rho_{2}^{\prime} \geq \ldots \geq \rho_{m}^{\prime}$ (where $m:=\lceil\lambda n\rceil$ ). (There will be no confusion with the semi-axes of the ellipsoid of maximal volume since we do not consider this ellipsoid in this proof.)

The natural Euclidean structure in $E$ is of course given by $P B_{2}=B_{2} \cap E$, and by the definition of $\mathcal{F}_{m}(K)$ we have $P B_{2} \subset C_{\lambda} P K$. On the other hand, clearly, $P K \subset P \mathcal{E}_{K}$, and hence $\rho_{m}^{\prime} \geq C_{\lambda}^{-1}$.

We first observe that since $E \in \mathcal{F}_{m}(K)$ then for all $1 \leq j \leq\lceil m / 2\rceil$ we have

$$
\rho_{j}^{\prime} \leq \rho_{j} \leq\left(1 / b_{\lambda}\right) \rho_{j}^{\prime} .
$$

Indeed, given an ellipsoid $\mathcal{D} \subset \mathbb{R}^{m}$ with semi-axes $\lambda_{1} \geq \lambda_{2} \geq \ldots \geq \lambda_{m}$ one has

$$
\lambda_{j}=\inf _{L} \sup _{x \in L \cap \mathcal{D}}|x|,
$$

where infimum is taken over all $(m-j+1)$-dimensional subspaces $L$. Thus, since $|P x| \leq|x|$ for $x \in \mathbb{R}^{n}$, we have $\rho_{j}^{\prime} \leq \rho_{j}$ for every $j \leq m$. On the other hand, since $\lceil m / 2\rceil=\lceil\lambda n / 2\rceil$, by the definition of $\mathcal{F}_{m}(K)$, we have $|P x| \geq b_{\lambda}|x|$ for every $x \in E_{0}:=\operatorname{span}\left\{e_{i} \mid 1 \leq i \leq\lceil m / 2\rceil\right\}$, which means that the operator

$$
\left.P\right|_{E_{0}}:\left(E_{0}, B_{2} \cap E_{0}\right) \rightarrow\left(P E_{0}, P B_{2}\right)
$$

is invertible with the norm of the inverse bounded by $1 / b_{\lambda}$. That implies $\rho_{j} \leq\left(1 / b_{\lambda}\right) \rho_{j}^{\prime}$.

Now by $(4.2)$ we get

$$
\begin{aligned}
\left(\frac{\left|\mathcal{E}_{K}\right|}{|K|}\right)^{1 / n} & =\left(\frac{\left|\mathcal{E}_{K}\right|}{\left|B_{2}\right|}\right)^{1 / n}=\left(\prod_{i=1}^{n} \rho_{i}\right)^{1 / n} \leq\left(\prod_{i=1}^{\lceil m / 2\rceil} \rho_{i}\right)^{1 /\lceil m / 2\rceil} \\
& \leq \frac{C_{\lambda}}{b_{\lambda}}\left(\prod_{i=1}^{m} \rho_{i}^{\prime}\right)^{2 / m}=\frac{C_{\lambda}}{b_{\lambda}}\left(\frac{\left|P \mathcal{E}_{K}\right|}{\left|P B_{2}\right|}\right)^{2 / m}
\end{aligned}
$$

Let $\mathcal{D} \supset P K$ be the ellipsoid of minimal volume for $P K$, so that

$$
|\mathcal{D}|^{1 / m}=\operatorname{outvr}(P K)|P K|^{1 / m} .
$$


Now let us note that $\mathcal{D} \supset \operatorname{conv}\{P K,-P K\}$ and $\mathcal{E}_{K}$ is also the ellipsoid of minimal volume for conv $\{P K,-P K\}$. Applying Corollary 2.3 for the symmetric convex body conv $\{P K,-P K\}$ and the ellipsoids $\mathcal{E}=P \mathcal{E}_{K} \subset E$ and $\mathcal{D} \subset E$ we get, by (2.2),

$$
\left|P \mathcal{E}_{K}\right|^{1 / m} \leq(c / \sqrt{\lambda})|\mathcal{D}|^{1 / m}
$$

By the definition of $M$-ellipsoid we have $|P K| \leq \exp \left(C_{0} n\right)\left|P B_{2}\right|$. Thus we get

$$
\begin{aligned}
\left(\frac{\left|P \mathcal{E}_{K}\right|}{\left|P B_{2}\right|}\right)^{2 / m} & \leq\left(c^{2} / \lambda\right)(\operatorname{outvr}(P K))^{2}\left(\frac{|P K|}{\left|P B_{2}\right|}\right)^{2 / m} \\
& \leq\left(c^{2} \exp \left(2 C_{0} / \lambda\right) / \lambda\right)(\operatorname{outvr}(P K))^{2}
\end{aligned}
$$

Combining this with (4.3) and the form of $b_{\lambda}$ we obtain (4.1) with $C_{\lambda}^{\prime}=$ $\left(c^{2} / c_{0}\right) C_{\lambda} \lambda^{-3 / 2} \exp \left(2 C_{0} / \lambda\right)$, which completes the proof.

Now we are in the position to prove Theorem 4.1.

Proof of Theorem 4.1 By the definition of $M$-position (3.1) and by (3.2), we have

$$
|P K| \leq \exp \left(C_{0} n\right)\left|P B_{2}\right|
$$

for every projection $P$. Thus, by the definitions of $\mathcal{F}_{\lceil\lambda n\rceil}(K)$ we have that every quotient $q K \in \mathcal{F}_{q, \lambda n}(K)$ admits an estimate for the volume ratio,

$$
\operatorname{vr}(q K) \leq\left(\left|P_{E} K\right| /\left|C_{\lambda}^{-1} P_{E} B_{2}\right|\right)^{1 /\lceil\lambda n\rceil} \leq C_{\lambda} \exp \left(C_{0} / \lambda\right) .
$$

By the duality every subspace $s L \in \mathcal{F}_{s,\lceil\lambda n\rceil}(L)$ admits an estimate for the outer volume ratio, $\operatorname{outvr}(s L) \leq a_{\lambda}:=c C_{\lambda} \exp \left(C_{0} / \lambda\right)$, where $c$ is an absolute constant. Now, let $q K$ and $s L$ satisfy the hypothesis of the theorem, then

$$
\operatorname{outvr}(q K) \leq d \operatorname{outvr}(s L) \leq a_{\lambda} d .
$$

By Proposition 4.2 we obtain

$$
\operatorname{outvr}(K) \leq C_{\lambda}^{\prime}\left(a_{\lambda} d\right)^{2} .
$$

The estimate for $\operatorname{vr}(L)$ follows by duality. 
Remark Instead of using duality argument we could use entropy estimates, since, as we discussed above, they holds for bodies $K-K, K \cap(-K), L-L$, $L \cap(-L)$ as well.

Remark The dependence on $d$ in $C(\lambda, d)$ can be improved by using the remark after Proposition 4.2 and a modification of the family $\mathcal{F}_{\lceil\lambda n\rceil}(K)$. We then obtain that for every $\alpha>1, C(\lambda, d) \leq C_{\lambda, \alpha} d^{\alpha}$, where $C_{\lambda, \alpha}$ depends on $\lambda$ and $\alpha$ only.

Setting $K=B_{2}$ in Theorem 4.1 we get an interesting corollary.

Corollary 4.3 Let $L$ be a convex body in $\mathbb{R}^{n}$ in $M$-position. If for some $0<\lambda<1$ and some $d>1$ a random $\lceil\lambda n\rceil$ section $s L$ of $L$ is $d$-Euclidean, then $\operatorname{vr}(L) \leq C$, where $C=C(\lambda, d)$ is a function of $\lambda$ and $d$ only.

This corollary was proved in [MS2] in the case when $L=-L$ and the Euclidean distance was replaced by the geometric distance to the ball $B_{2}$. In this case it is shown by combining Theorems $3.1^{\prime}$ and 2.2 in [MS2], that for any $0<\xi<1$, a random section of $L$ is $C$-equivalent to $B_{2}$.

Theorem 4.1 has the following standard consequence about the existence of a large family of Euclidean quotients and subspaces.

Corollary 4.4 Let $K$ and $L$ be two convex bodies in $\mathbb{R}^{n}$ in $M$-position, and assume that for some $0<\lambda<1$ and some $d>1$ there is a quotient space $q K \in \mathcal{F}_{q,\lceil\lambda n\rceil}(K)$ and a subspace $s L \in \mathcal{F}_{s,\lceil\lambda n\rceil}(L)$ such that the Banach-Mazur distance satisfies

$$
d(q K, s L) \leq d
$$

Then for every $0<\xi<1$ a random orthogonal (in $\mathcal{E}_{K}$ ) projection of $K$ is $\bar{C}$-Euclidean and a random (in $\mathcal{E}_{L}^{\prime}$ ) section of $L$ is $\bar{C}$-Euclidean, where $\bar{C}=\bar{C}(\lambda, \xi, d)$ is a function of $\lambda, \xi$ and $d$ only.

Proof The proof relies on the volume ratio argument. Recall that since $\mathcal{E}_{K}$ is the ellipsoid of minimal volume for $K$ then a random orthogonal (in $\mathcal{E}_{K}$ ) projection satisfies

$$
P_{E} \mathcal{E}_{K} \subset(4 \pi \text { outvr }(K))^{1 /(1-\lambda)} P_{E} K
$$

and since $\mathcal{E}_{L}^{\prime}$ is the ellipsoid of maximal volume for $L$ then a random (in $\mathcal{E}_{L}^{\prime}$ ) subspace $E$ of $\mathbb{R}^{n}$ satisfies

$$
K \cap E \subset(4 \pi \operatorname{vr}(K))^{1 /(1-\lambda)} \mathcal{E}_{K}^{\prime} \cap E
$$


(see e.g. Chapter 6 of [Pi] and note that the proof doesn't require the symmetry).

The conclusion of the corollary follows directly from Theorem 4.1 and (4.5), (4.6) with $\bar{C}=(4 \pi C)^{1 /(1-\xi)}$, where $C$ is a function from Theorem 4.1.

As we have just seen, the closeness of spaces $q K$ and $s L$ in Theorem 4.1 and Corollary 4.4 implies the existence of many Euclidean quotients and subspaces, of an arbitrary proportional dimension, for $K$ and $L$, respectively. However one may ask whether spaces $q K$ and $s L$ themselves are isomorphic to Euclidean as well? Surprisingly, the answer in general is no even in the symmetric case. An example in [LMT] shows that for some symmetric convex bodies $K$ and $L$ one may select $M$-ellipsoids in such a way that random quotients of $K$ and subspaces of $L$ are far from Euclidean, while being close together. At the same time we believe that it might be true that for a judiciously selected $M$-ellipsoid, the hypothesis of our theorem indeed implies that $q K$ and $s L$ are Euclidean, with a high probability.

Similar as in [LMT], we now pass to a discussion of the global form of the results of the first part of this section. Although we always have an analogy between local and global results, there is no an abstract argument proving this. In the present context the global result is much easier.

Instead of working with random families of subspaces of $\mathbb{R}^{n}$ we will work with random families of orthogonal operators. Let $O(n)$ denote the group of orthogonal operators on $\mathbb{R}^{n}$ and let $\nu$ denote the normalized Haar measure on $O(n)$. Given a convex body $K \subset \mathbb{R}^{n}$ in $M$-position define $\mathcal{H}(K)$ as the set of all operators $U \in O(n)$ satisfying

(i) $c B_{2} \subset K+U K$,

(ii) $c(K \cap U K) \subset B_{2}$

for some absolute constant $c>0$. These two conditions are the global form of the conditions (i) and (ii) in the definition of the random family $\mathcal{F}_{[\lambda n]}(K)$. It can be shown that there exists a choice of $c>0$ such that

$$
\nu(\mathcal{H}(K)) \geq 1-e^{-c_{1} n},
$$

where $c_{1}>0$ is an absolute constant (see [M5] for the symmetric case and [MPa2] (and also Theorem $2^{\prime}$ of [LMP]) for the non-symmetric case). 
Note that $(K+U K)^{0}$ is 2-equivalent to $K^{0} \cap\left(U^{*}\right)^{-1} K^{0}$ and $(K \cap U K)^{0}$ is 2-equivalent to $K^{0}+\left(U^{*}\right)^{-1} K^{0}$. Thus, since $\left(U^{*}\right)^{-1}=U$ for $U \in O(n)$, we obtain that $\mathcal{H}(K)=\mathcal{H}\left(K^{0}\right)$, possibly replacing the constant $c$ in the definition by $c / 2$.

The following theorem is the global version of Theorem 4.1.

Theorem 4.5 Let $K$ and $L$ be two convex bodies in $\mathbb{R}^{n}$ in $M$-position. Assume that there are operators $U \in \mathcal{H}(K), V \in \mathcal{H}(L)$, and some $d>1$ such that

$$
d\left(K_{0}, L_{0}\right) \leq d,
$$

where $K_{0}=K+U K$ and $L_{0}=L \cap V L$. Then

$$
\operatorname{outvr}(K) \leq\left(C_{1} / c\right) \exp \left(4 C_{0}\right) d \quad \text { and } \quad \operatorname{vr}(L) \leq\left(C_{1} / c\right) \exp \left(4 C_{0}\right) d
$$

where $C_{1}$ is an absolute constant and $c$ is from the definition of the families $\mathcal{H}(K)$ and $\mathcal{H}(L)$.

Proof By the definition of the set $\mathcal{H}(K)$ we have $c B_{2} \subset K_{0}$. On the other hand, by the definition of $M$-ellipsoid and covering numbers we obtain that $K+U K$ can be covered by $\exp \left(2 C_{0} n\right)$ translations of $2 B_{2}$. That implies

$$
\operatorname{vr}\left(K_{0}\right) \leq\left(\left|K_{0}\right| /\left|c B_{2}\right|\right)^{1 / n} \leq(2 / c) \exp \left(2 C_{0}\right) .
$$

To find the upper bound for the outer volume ratio of $L_{0}$ we are going to use duality. Indeed, $c B_{2} \subset L_{0}^{0}=\operatorname{conv}\left(L_{0}, V L_{0}\right) \subset L^{0}+V L_{0}$. Thus repeating the proof above and using Bourgain-Milman's inverse Santaló inequality ([BM2]) we obtain

$$
\operatorname{outvr}\left(L_{0}\right) \leq\left(\left|(1 / c) B_{2}\right| /\left|L_{0}\right|\right)^{1 / n} \leq C_{1}\left(\left|L_{0}^{0}\right| /\left|c B_{2}\right|\right)^{1 / n} \leq\left(2 C_{1} / c\right) \exp \left(2 C_{0}\right)
$$

where $C_{1}>0$ is an absolute constant. Since $d\left(K_{0}, L_{0}\right) \leq d$, then $K_{0}$ has outer volume ratio bounded by $\left(2 C_{1} d / c\right) \exp \left(2 C_{0}\right)$. Let $\mathcal{E}$ be the minimal volume ellipsoid for $K_{0}$. Then $K \subset K_{0} \subset \mathcal{E}$ and

$$
\left(\frac{|\mathcal{E}|}{|K|}\right)^{1 / n}=\left(\frac{|\mathcal{E}|}{\left|K_{0}\right|}\right)^{1 / n}\left(\frac{\left|K_{0}\right|}{\left|B_{2}\right|}\right)^{1 / n}\left(\frac{\left|B_{2}\right|}{|K|}\right)^{1 / n} \leq\left(4 C_{1} / c\right) \exp \left(2 C_{0}\right) d,
$$

which implies boundedness of outvr $(K)$. The result for vr $L$ follows by the similar argument. 
Remark It is clear from the proof that the theorem can be generalized to the case of many orthogonal operators. Namely, let $K, L, U$, and $V$ be as in the theorem. Assume further that $U_{1}, \ldots, U_{k}$ and $V_{1}, \ldots, V_{m}$ are arbitrary orthogonal operators on $\mathbb{R}^{n}$. Let $K_{0}=K+U K+U_{1} K+\ldots+U_{k} K$ and $L_{0}=L \cap V L \cap V_{1} L \cap \ldots \cap V_{m} L$. Then if $d\left(K_{0}, L_{0}\right) \leq d$ then

$$
\operatorname{outvr}(K) \leq C d \quad \text { and } \quad \operatorname{vr}(L) \leq C d
$$

where $C$ is a function of $k, m, c$ and $C_{0}$ only.

\section{Technical remarks and improvements}

In this section we discuss possible improvements of our results and of dependence of functions and constant on parameters.

First we would like to note that in the symmetric case, choosing a stronger definition of an $M$-ellipsoid, the dependence of $C_{\lambda}$ on $\lambda$ in the definition of our random family $\mathcal{F}_{\lceil\lambda n\rceil}(K)$ can be improved to a polynomial dependence on $1 /(1-\lambda)$. Indeed, for every $\varepsilon>0$ there exists an ellipsoid $D$ such that

$$
\begin{aligned}
\max \{N(K, t D), N & \left.(D, t K), N\left(K^{0}, t D^{0}\right), N\left(D^{0}, t K^{0}\right)\right\} \\
& \leq \exp \left(n\left(C_{\varepsilon} / t\right)^{\alpha(\varepsilon)}\right)
\end{aligned}
$$

for $\alpha(\varepsilon)=(\varepsilon+1 / 2)^{-1}$ and some $C_{\varepsilon}$ depending on $\varepsilon$ only (see e.g. Corollary 7.16 of $[\mathrm{Pi}])$. If we take such estimates as a definition of $M$-ellipsoid then repeating the proof of Theorem 3 in [LMP] (cf. the proof of Proposition $7^{\prime}$ of [MPi]), one can obtain $C_{\lambda} \leq f(\varepsilon)(1-\lambda)^{-(1+\varepsilon)}$. Moreover, if we use the definition of $M$-ellipsoid given by Theorem 7.13 of [Pi] then, applying Theorem 3.2 of [LT], we immediately observe that $C_{\lambda} \leq C \varepsilon^{-3 / 2}(1-\lambda)^{-(\varepsilon+1 / 2)}$.

Now we pass to the discussion related to Theorem 4.1 and Proposition 4.2 and their consequences.

We start with Proposition 4.2. It says in qualitative terms that for a body $K$ in $M$-position, if outvr $\left(P_{E} K\right)$ is small for a random projection $P_{E}$, then outvr $(K)$ is small. As was noted after the statement of this proposition the power 2 in estimate (4.1) can be improved to any $\alpha>1$. This follows from the proof. Namely, fix $\alpha>1$; then for any $0<\lambda<1$ we can define a family $\mathcal{F}_{\lceil\lambda n\rceil}^{(\alpha)}(K)$ satisfying measure estimate depending additionally on $\alpha$ such that for any $q K \in \mathcal{F}_{q,\lceil\lambda n\rceil}^{(\alpha)}(K)$ we have outvr $(K) \leq C_{\lambda, \alpha}^{\prime}(\operatorname{outvr}(q K))^{\alpha}$, where $C_{\lambda, \alpha}^{\prime}$ depends on $\lambda$ and $\alpha$ only. 
Using the same idea as in the proof of Proposition 4.2 we can show that in the situation from the proposition one can get the inclusion $Q K \subset C^{\prime \prime} Q B_{2}$, for a certain projection $Q$ of an arbitrarily large proportional dimension. In fact we get more.

Proposition 5.1 Under the assumptions of Proposition 4.2 for every $0<$ $\xi<1$ there exists a projection $Q$ with rank $Q \geq \xi n$, orthogonal in the standard Euclidean structure and in $\mathcal{E}_{K}$, such that $Q \mathcal{E}_{K} \subset C^{\prime \prime} Q B_{2}$ where

$$
C^{\prime \prime}=C_{\lambda}^{-1}\left(\frac{c}{c_{0}} \frac{C_{\lambda}}{\lambda^{3 / 2}} \exp \left(2 C_{0} / \lambda\right)\right)^{\beta}\left(\operatorname{outvr}\left(P_{E} K\right)\right)^{2 \beta},
$$

with $\beta=\max \{1,\lceil\lambda n / 2\rceil /\lceil(1-\xi) n\rceil\} \leq 1+\lambda / 2(1-\xi)$, and $c>0$ is an absolute constant. In particular, $Q K \subset C^{\prime \prime} Q B_{2}$.

Proof We use the same setting and notations as in the proof of Proposition 4.2 .

Let us note that the proof of the Proposition also gives

$$
\left(\prod_{i=1}^{\lceil m / 2\rceil} \rho_{i}\right)^{1 /\lceil m / 2\rceil} \leq A:=\frac{c^{2} C_{\lambda}}{c_{0} \lambda^{3 / 2}} \exp \left(2 C_{0} / \lambda\right)(\operatorname{outvr}(P K))^{2}
$$

and $\rho_{m / 2} \geq 1 / C_{\lambda}$.

Since for every $1 \leq k \leq\lceil m / 2\rceil$ we have

$$
\prod_{i=1}^{\lceil m / 2\rceil} \rho_{i} \geq \rho_{k}^{k} \cdot \rho_{\lceil m / 2\rceil}^{\lceil m / 2\rceil-k}
$$

we obtain $\rho_{k} \leq C_{\lambda}^{-1}\left(C_{\lambda} A\right)^{\lceil m / 2\rceil / k}$, for every $1 \leq k \leq\lceil m / 2\rceil$.

Now let $0<\xi<1$ and let $k_{0}=\lceil(1-\xi) n\rceil$. If $k_{0} \geq\lceil m / 2\rceil$ then we have $\rho_{k_{0}} \leq \rho_{\lceil m / 2\rceil} \leq A$. If $k_{0}<\lceil m / 2\rceil$ then $\rho_{k_{0}} \leq C_{\lambda}^{-1}\left(C_{\lambda} A\right)^{\lceil m / 2\rceil / k_{0}}$. Let $Q$ be the orthogonal projection onto span $\left\{e_{i} \mid k_{0} \leq i \leq n\right\}$, that is, $Q$ "kills" the $k_{0}-1$ largest semiaxes of $\mathcal{E}_{K}$. Then we obtain

$$
Q \mathcal{E}_{K} \subset C_{\lambda}^{-1}\left(C_{\lambda} A\right)^{\beta} Q B_{2},
$$

where $\beta=\lceil m / 2\rceil /\lceil(1-\xi) n\rceil$ if $\lceil(1-\xi) n\rceil<\lceil m / 2\rceil$ and $\beta=1$ otherwise. Since $\lceil\lambda n / 2\rceil=\lceil m / 2\rceil$ we obtain the result. 
We turn now to Theorem 4.1.

First let us comment on dependence of $C$ on $d$ in this theorem. The proof we presented gives $C$ of the form $C=C(\lambda, d) \leq C_{\lambda}^{\prime \prime} d^{2}$, where $C_{\lambda}^{\prime \prime}$ depends on $\lambda$ only. Using Remark after Proposition 4.2 and discussion before Proposition 5.1 we can obtain that for every $\alpha>1$, this dependence can be improved to $C_{\lambda, \alpha}^{\prime \prime} d^{\alpha}$, where $C_{\lambda, \alpha}^{\prime \prime}$ depends on $\lambda$ and $\alpha$ only.

Let us also note that, as can be seen from the proof, the theorem holds even for bodies of different dimensions. Namely, the following theorem holds.

Theorem 5.2 Let $K \subset \mathbb{R}^{n}$ and $L \subset \mathbb{R}^{m}$ be two symmetric convex bodies in $M$-position. Let $1 \leq k<\min (n, m)$ and assume that for some quotient space $q K \in \mathcal{F}_{q, k}(K)$ and some subspace $s L \in \mathcal{F}_{s, k}(L)$ one has $d(q K, s L) \leq d$. Then outvr $(K) \leq C$ and $\operatorname{vr}(L) \leq C$, where $C=C(k / n, k / m, d)$.

Since Corollary 4.4 follows immediately from Theorem 4.1 we can also extend Corollary 4.4 to the case of different dimensions of $K$ and $L$. Namely, we have the following corollary of Theorem 5.2.

Corollary 5.3 Let $K \subset \mathbb{R}^{n}$ and $L \subset \mathbb{R}^{m}$ be two symmetric convex bodies in $M$-position. Let $1 \leq k<\min (n, m)$ and assume that for some quotient space $q K \in \mathcal{F}_{q, k}(K)$ and some subspace $s L \in \mathcal{F}_{s, k}(L)$ one has $d(q K, s L) \leq d$. Then for every $0<\xi<1$ a random orthogonal (in $\mathcal{E}_{K}$ ) projection of $K$ is $\bar{C}$-Euclidean and a random (in $\mathcal{E}_{L}^{\prime}$ ) section of $L$ is $\bar{C}$-Euclidean, where $\bar{C}=\bar{C}(\lambda, \xi, d)$ is a function of $\lambda, \xi$ and $d$ only.

Remarks 1. In the situation of Corollaries 4.4 and 5.3, it follows from the proof that in fact a random projection $Q K$ of $K$ is $C$-equivalent to $Q \mathcal{E}_{K}$. Similarly, a random section $L \cap E$ of $L$ is $C$-equivalent to $\mathcal{E}_{L}^{\prime} \cap E$.

2. In the situation of Theorems 4.1 and 5.2 we also have that for every $0<\xi<1$, there exists a projection $Q$ with rank $P \geq \xi n$, orthogonal in the standard Euclidean structure and in $\mathcal{E}_{K}$, such that $Q \mathcal{E}_{K} \subset C^{\prime} Q B_{2}$; and similarly, there exists a subspace $F \subset \mathbb{R}^{n}$ with $\operatorname{dim} F \geq \xi n$ such that $\mathcal{E}_{L}^{\prime} \cap F \supset$ $\left(1 / C^{\prime}\right) B_{2} \cap F$. Here $C^{\prime}=C^{\prime}(\lambda, \xi, d)$ is a function of $\lambda, \xi$ and $d$. This follows from Proposition 5.1 above. 


\section{The symmetric quasi-convex case}

Here we discuss briefly the symmetric quasi-convex cases.

Let $C \geq 1$. Recall that a body $K$ is called $C$-quasi-convex if $\alpha K+(1-$ $\alpha) K \subset C K$ for every $\alpha \in[0,1]$. Given $p \in(0,1]$ we say that a body $K$ is $p$-convex if $\alpha K+\left(1-\alpha^{p}\right)^{1 / p} K \subset K$ for every $\alpha \in[0,1]$. Note that if $C=1$ or $p=1$ then the definitions above give a convex body. Clearly every $p$-convex symmetric body is $C$-quasi-convex with $2 C=2^{1 / p}$. By AokiRolewicz theorem (see $[\mathrm{KPR}],[\mathrm{Ko}],[\mathrm{Ro}]$ ) every $C$-quasi-convex body is $2 C$ equivalent to some $p$-convex body, where $p$ satisfies $2 C=2^{1 / p}$. Hence it is enough to restrict ourselves to a $p$-convex case. Note that the gauge of a $p$-convex body satisfies $\|x+y\|^{p} \leq\|x\|^{p}+\|y\|^{p}$. It should be also noted that since $K^{0}=(\operatorname{conv} K)^{0}$, duality arguments usually lead to very weak results for $p$-convex bodies. Thus to extend proofs which originally used duality, we need to find direct arguments.

We say that $p$-convex body $K$ is in $M$-position if there exists $q>0$ and $C_{p}$, depending on $p$ and $q$ only, such that

$$
\max \left\{N\left(B_{2}, t K\right), N\left(K, t B_{2}\right)\right\} \leq \exp \left(n\left(C_{p} / t\right)^{q}\right)
$$

for every $t \geq 1$. The existence of $M$-position for every symmetric $p$-convex body and every $q<(2 p) /(2-p)$ was proved in [BBP] (see also [L] for the dependence of $C_{p}$ on $p$ and $q$ ). We can assume, as we did before, that a body $K$ in $M$-position satisfies $|K|=\left|B_{2}\right|$. We will now show that all theorems of Section 3 can be extended to the $p$-convex setting. Of course all constants appearing in the statements will depend on $p$.

It was shown in $[\mathrm{LMP}]$ that if $p$-convex body satisfies entropy estimates (6.1) then the set of $\lceil\lambda n\rceil$-dimensional subspaces $E$ of $\mathbb{R}^{n}$ satisfying

$$
P_{E} B_{2} \subset(1-\lambda)^{-1-1 / p}(c / p)^{c / p} P_{E} K
$$

and

$$
K \cap E \subset(1-\lambda)^{-1-1 / p}(c / p)^{c / p} B_{2} \cap E
$$

for some absolute constant $c>0$, has measure exponentially close to 1 . This corresponds to the conditions (i) and (ii) of the definition of random family. (Actually only the existence of such a subspace was stated explicitely, but the measure estimates follow from the proof.) This allows us to define, for every symmetric $p$-convex body $K$, a random family $\mathcal{F}_{\lceil\lambda n\rceil}(K)$ in the same 
way as we did before. Note in passing that a maximal volume ellipsoid might not be unique in the $p$-convex case, so before defining the random family we fix one of the maximal volume ellipsoids for the rest of the argument.

Since the arguments in Section 3 several times use duality, we need to introduce a new property $(* *)$, which plays a role of a dual property to $(*)$. We say that an ellipsoid $\mathcal{E} \in \mathbb{R}^{m}$ has property ( $\left.* *\right)$ with respect to a $p$-convex body $K \in \mathbb{R}^{m}$ with constant $a \geq 1$ if

(**) for any $1 \leq k \leq m$ and any $k$-dimensional subspace $E \subset \mathbb{R}^{m}$ we have

$$
\left\|i_{\mid E}: \mathcal{E} \cap E \rightarrow K\right\| \geq(1 / a)(k / m)^{1 / p-1 / 2},
$$

where $i_{\mid E}$ is the identity map on $E$.

Note that for simplicity we restrict ourselves to the case of function $\phi(t)=$ $t^{1 / p-1 / 2}$.

Theorem 2.1 is stated for ellipsoids, so we can dualize it. We then get

Theorem 6.1 Let $\mathcal{E} \subset \mathbb{R}^{m}$ and $\mathcal{D} \subset \mathbb{R}^{m}$ be two ellipsoids, let $B:=\operatorname{conv}(\mathcal{E}, \mathcal{D})$ and assume that $\mathcal{E}$ has property (**) with constant $a \geq 1$ with respect to $B$. Then

$$
|\mathcal{D}|^{1 / m} \leq c^{1 / p} a|\mathcal{E}|^{1 / m}
$$

where $c>0$ is an absolute constant.

The following lemma is the dual version of Lemma 2.2.

Lemma 6.2 Let $p \in(0,1), K$ be a symmetric $p$-convex body and $\mathcal{E}$ be an ellipsoid of maximal volume for $K$. Let $F$ be an an-dimensional subspace of $\mathbb{R}^{n}$. Then $\mathcal{E} \cap F$ has property (**) with respect to $K \cap F$ with constant $a=(e / \alpha)^{1 / p-1 / 2}$

We postpone the proof of this lemma until the end of this section.

Now we will show that Proposition 4.2 as well as its dual form are valid in the $p$-convex case. Namely, the following statement holds.

Proposition 6.3 Let $p \in(0,1), K \subset \mathbb{R}^{n}$ be a symmetric $p$-convex body in $M$-position. Let $0<\lambda<1$. Let $E \in \mathcal{F}_{\lceil\lambda n\rceil}(K)$ and let $P_{E}$ be the orthogonal projection on $E$. Then

$$
\operatorname{outvr}(K) \leq C\left(\operatorname{outvr}\left(P_{E} K\right)\right)^{2} \quad \text { and } \quad \operatorname{vr}(K) \leq C(\operatorname{vr}(K \cap E))^{2},
$$

where $C$ depends on $\lambda$ and $p$ only. 
Remark Of course the power 2 can be improved the same way as in the convex case.

Proof The case outvr $(K)$ repeats the proof of Proposition 4.2, using the observation that the ellipsoid of minimal volume for a $p$-convex symmetric body $K$ is the ellipsoid of minimal volume for conv $K$ as well.

To prove the estimate for $\operatorname{vr}(K)$ we observe that the same ideas (with obvious modifications) as were used in the "convex" proof before (4.4) imply for $E \subset \mathcal{F}_{\lceil\lambda n\rceil}$

$$
\left(|K| /\left|c E_{K}^{\prime}\right|\right)^{1 / n} \leq C(\lambda, p) b_{\lambda}^{-1}\left(\left|B_{2} \cap E\right| /\left|\mathcal{E}_{K}^{\prime} \cap E\right|\right)^{2 / m},
$$

where $\mathcal{E}_{K}^{\prime}$ is the ellipsoid of maximal volume for $K$ which was fixed before the definition of the random family. Now let $\mathcal{D} \subset K \cap E$ be an ellipsoid of maximal volume for $K \cap E$ and let $\mathcal{E}=\mathcal{E}_{K}^{\prime} \cap E$. Then, by Lemma 6.2, the ellipsoid $\mathcal{E}$ has property (**) with respect to $K \cap E$ with constant $a=$ $(e / \lambda)^{1 / p-1 / 2}$. Since $K$ is $p$-convex, then $\operatorname{conv}(\mathcal{D}, \mathcal{E}) \subset 2^{-1+1 / p} K$. Hence, $\mathcal{E}$ has property $(* *)$ with respect to $\operatorname{conv}(\mathcal{D}, \mathcal{E})$ with constant $a=(2 e / \lambda)^{1 / p-1 / 2}$. Therefore, by Theorem 6.1 we obtain $|\mathcal{D}|^{1 / m} \leq c_{p} a|\mathcal{E}|^{1 / m}$, which implies

$$
\begin{aligned}
\left(\left|B_{2} \cap E\right| /|\mathcal{E}|\right)^{2 / m} & \leq c_{p}^{2} a^{2}\left(\left|B_{2} \cap E\right| /|\mathcal{D}|\right)^{2 / m} \\
& =c_{p}^{2} a^{2}(\operatorname{vr}(K \cap E))^{2}\left(\left|B_{2} \cap E\right| /|K \cap E|\right)^{2 / m} .
\end{aligned}
$$

The proposition now follows by covering estimates and (3.2) (note that $K=$ $-K$ and $\left.K-K \subset 2^{1 / p} K\right)$.

Now we can prove our main result, Theorem 4.1, for symmetric $p$-convex bodies.

Theorem 6.4 Let $p \in(0,1), K$ and $L$ be two symmetric $p$-convex bodies in $\mathbb{R}^{n}$ in $M$-position, and assume that for some $0<\lambda<1$ and some $d>1$ there is a quotient space $q K \in \mathcal{F}_{q,\lceil\lambda n\rceil}(K)$ and a subspace $s L \in \mathcal{F}_{s,\lceil\lambda n\rceil}(L)$ such that the Banach-Mazur distance satisfies

$$
d(q K, s L) \leq d
$$

Then

$$
\operatorname{outvr}(K) \leq C \quad \text { and } \quad \operatorname{vr}(L) \leq C,
$$

where $C=C(\lambda, d, p)$ is a function of $\lambda, d$ and $p$ only. 
As in the convex case the proof is based on the previous proposition.

Proof By the definition of $M$-position (6.1) and by (3.2), we have

$$
|P K| \leq \exp \left(C_{0} n\right)\left|P B_{2}\right| \quad \text { and } \quad\left|B_{2} \cap E\right| \leq \exp \left(C_{0} n\right)\left|2^{1 / p} L \cap E\right|
$$

for every projection $P$ and every subspace $E$ (we used again that $L-L \subset$ $\left.2^{1 / p} L\right)$. Thus, by the definitions of $\mathcal{F}_{\lceil\lambda n\rceil}(K)$ we have that every quotient $q K \in \mathcal{F}_{q, \lambda n}(K)$ admits an estimate for the volume ratio,

$$
\operatorname{vr}(q K) \leq\left(\left|P_{E} K\right| /\left|C_{\lambda}^{-1} P_{E} B_{2}\right|\right)^{1 /\lceil\lambda n\rceil} \leq C_{\lambda} \exp \left(C_{0} / \lambda\right) .
$$

Similarly, every subspace $s L \in \mathcal{F}_{s,\lceil\lambda n\rceil}(L)$ admits an estimate for the outer volume ratio, outvr $(s L) \leq a_{\lambda}:=2^{1 / p} C_{\lambda} \exp \left(C_{0} / \lambda\right)$. Now, let $q K$ and $s L$ satisfy the hypothesis of the theorem, then

$$
\operatorname{outvr}(q K) \leq d \operatorname{outvr}(s L) \leq a_{\lambda} d \quad \text { and } \quad \operatorname{vr}(s L) \leq \operatorname{vr}(q K) \leq a_{\lambda} d .
$$

By Proposition 6.3 we obtain

$$
\operatorname{outvr}(K) \leq C_{\lambda}^{\prime}\left(a_{\lambda} d\right)^{2} \quad \text { and } \quad \operatorname{vr}(L) \leq C_{\lambda}^{\prime}\left(a_{\lambda} d\right)^{2} .
$$

Corollary 4.4 in the $p$-convex case follows from Theorem 6.4. Indeed, it is not difficult to check that volume ratio argument works for $p$-convex bodies as well (with constants depending on $p$ ). If $p$-convex symmetric body $K$ has bounded outer volume ratio then so is conv $K$. Thus, by corresponding "convex" result $P$ conv $K=\operatorname{conv} P K$ is $C$-Euclidean. Applying results from $[\mathrm{GK}]$ (Lemma 3 and Remark that follows) we obtain that $P K$ itself is $C(p)$-Euclidean (cf. proof of Theorem 13 in $[\mathrm{KT}]$ )).

To prove the global results let us note first that the existence of othogonal operator $U$ satisfying conditions (i) and (ii) of section 4 was proved in [M6] (see also Lemmas 6.3 and 6.8 of [LMS] and Theorem $2^{\prime}$ of [LMP]). Moreover, it follows from this proof that measure of such operators is exponentially close to 1 . This means that we can define the family of operators $\mathcal{H}(K)$ the same way as before. The only place in the proof of Theorem 4.5, where convexity was used is the estimate for outvr $L_{0}$. The necessary estimate in p-convex case follows from Lemma 6.4 of [LMS].

Finally, to prove Lemma 6.2, we prove the following lemma which obviously implies it. 
Lemma 6.5 Let $K$ be a symmetric p-convex body and $\mathcal{E}$ be an ellipsoid of maximal volume for $K$. Let $E$ be a $k$-dimensional subspace of $\mathbb{R}^{n}$. Then

$$
\left\|i_{\mid E}: \mathcal{E} \cap E \rightarrow K\right\| \geq(k / e n)^{1 / p-1 / 2} .
$$

Proof Without loss of generality we assume that $\mathcal{E}=B_{2}$ and $k<n$. Denote $B_{2}^{k}=B_{2} \cap \mathbb{R}^{k}$. Clearly it is enough to show that

$$
\left\|i_{\mid \mathbb{R}^{k}}: B_{2}^{k} \rightarrow K\right\| \leq 1 / A
$$

implies $A \leq(e n / k)^{1 / p-1 / 2}$. In other words, $A B_{2}^{k} \subset K$ implies $A \leq(e n / k)^{1 / p-1 / 2}$.

Assume $A B_{2}^{k} \subset K$. Set $1 / r=1 / p-1 / 2$. Let $b=A(k / n)^{1 / r}$ and $a=$ $(1-k / n)^{-1 / r}>1$. Consider the ellipsoid

$$
\mathcal{D}=\left\{x \in \mathbb{R}^{n} \mid \sum_{i=1}^{k} x_{i}^{2} / b^{2}+\sum_{i=k+1}^{n} x_{i}^{2} a^{2} \leq 1\right\} .
$$

We shall show that

$$
\mathcal{D} \subset L:=\bigcup_{\alpha^{p}+\beta^{p} \leq 1}\left(\alpha B_{2}+A \beta B_{2}^{k}\right)
$$

Since $B_{2} \subset K, A B_{2}^{k} \subset K$, by $p$-convexity of $K$, it will imply that $\mathcal{D} \subset K$.

Let $x \in \mathcal{D}$. Set $\gamma_{1}=\left(\sum_{i=1}^{k} x_{i}^{2}\right)^{1 / 2}$ and $\gamma_{2}=\left(\sum_{i=k+1}^{n} x_{i}^{2}\right)^{1 / 2}$.

Put $\alpha=\gamma_{2}$ and $\beta=\gamma_{1} / A$. Then we have $x=\alpha y+\beta z$ where $y=$ $\left(1 / \gamma_{2}\right)\left(0, \ldots, 0, x_{k+1}, \ldots, x_{n}\right) \in B_{2}$ and $z=\left(A / \gamma_{1}\right)\left(x_{1}, \ldots, x_{k}\right) \in A B_{2}^{k}$. Furthermore,

$$
\alpha^{p}+\beta^{p}=\left(\frac{1}{a}\left(\gamma_{2} a\right)\right)^{p}+\left(\frac{b}{A}\left(\gamma_{1} / b\right)\right)^{p} .
$$

Note that since $x \in \mathcal{D}$ then

$$
\left(\gamma_{2} a\right)^{2}+\left(\gamma_{1} / b\right)^{2} \leq 1
$$

So now, using Hölder's inequality for $1 / p=1 / r+1 / 2$ we get that $\alpha^{p}+\beta^{p}$ is less than or equal to

$$
\left(\frac{1}{a}\right)^{r}+\left(\frac{b}{A}\right)^{r}=1
$$

by definitions of $a$ and $b$. 
Thus $\mathcal{D} \subset K$. Now we compare the volumes of $\mathcal{D}$ and $B_{2}$ (recall that $B_{2}$ is an ellipsoid of maximal volume for $K$ ).

$$
|\mathcal{D}| /\left|B_{2}\right|=b^{k} a^{k-n}=\left(A^{r} k / n\right)^{k / r}(1-k / n)^{(n-k) / r} \geq\left(A^{r} k / n\right)^{k / r} e^{-k / r}>1
$$

if $A^{r}>e n / k$. This contradicts the maximality of the volume of $B_{2}$ and hence completes the proof.

Remark In the convex case $p=1$ a similar argument gives the best possible result

$$
\left\|i_{\mid E}: \mathcal{E} \cap E \rightarrow K\right\| \geq \sqrt{k / n}
$$

Indeed, with the same notations as above, assume that $A>\sqrt{k / n}$. Consider the ellipsoid $\mathcal{D}$, defined as before, for parameters $b=A \sqrt{k / n}$ and $a=\sqrt{\left(A^{2}-1\right) /\left(A^{2}-b^{2}\right)}$. Let $L:=\operatorname{conv}\left(B_{2}, A B_{2}^{k}\right) \subset K$. The direct computations shows that $\mathcal{D}^{0} \supset L^{0}=B_{2} \cap(1 / A) B_{2}^{k}$. Thus $\mathcal{D} \subset K$ and

$$
\left(|\mathcal{D}| /\left|B_{2}\right|\right)^{2}=\left(b^{k} a^{k-n}\right)^{2}=\left(A^{2} k / n\right)^{k}\left(\frac{1-k / n}{1-1 / A^{2}}\right)^{n-k}>1,
$$

since $A>\sqrt{n / k}$.

\section{References}

[BBP] J. Bastero, J. Bernués, J. Peña, An extension of Milman's reverse BrunnMinkowski inequality. Geom. Funct. Anal. 5 (1995), no. 3, 572-581.

[BM1] J. Bourgain, V. D. Milman, Distances between normed spaces, their subspaces and quotient spaces. Integral Equations Operator Theory 9 (1986), $31-46$.

[BM2] J. Bourgain, V. D. Milman, New volume ratio properties for symmetric bodies in $\mathbb{R}^{n}$, Invent. Math. 88 (1987), no 2, 319-340.

[D] S.J. Dilworth, The dimension of Euclidean subspaces of quasi-normed spaces, Math. Proc. Camb. Phil. Soc., 97 (1985), 311-320.

[GM] A. Giannopoulos, V. D. Milman, Euclidean structure in finite-dimensional normed spaces, In: Handbook of the geometry of Banach spaces, Vol. I, North Holland, Amsterdam, 2001, 707-779. 
[Gl] E. D. Gluskin, The diameter of Minkowski compactum roughly equals to n. Funct. Anal. Appl., 15 (1981), 57-58 (English translation).

[G] Y. Gordon, On Milman's inequality and random subspaces which escape through a mesh in $\mathbb{R}$. In: Geometric aspects of functional analysis (1986/87), 84-106, Lecture Notes in Math., 1317, Springer, Berlin-New York, 1988.

[GK] Y. Gordon and N.J. Kalton, Local structure theory for quasi-normed spaces. Bull. Sci. Math., 118 (1994), 441-453.

[KPR] N.J. Kalton, N.T. Peck and J.W. Roberts, An F-space sampler. London Mathematical Society Lecture Note Series, 89, Cambridge University Press, Cambridge-New-York, 1984.

[KT] N.J. Kalton and S. Tam, Factorization theorems for quasi-normed spaces Houston J. Math., 19 (1993), 301-317.

[Ko] H. König, Eigenvalue distribution of compact operators. Operator Theory: Advances and Applications, 16. Birkhäuser Verlag, Basel-Boston, Mass., 1986.

[L] A. E. Litvak, On the constant in the reverse Brunn-Minkowski inequality for p-convex balls, Convex Geometric Analysis, (Berkeley, CA, 1996), 129137, Math. Sci. Res. Inst. Publ. V. 34, Cambridge Univ. Press, Cambridge, 1999.

[LMP] A. E. Litvak, V. D. Milman, A. Pajor, The covering numbers and "low $M^{*}$-estimate" for quasi-convex bodies. Proc. Amer. Math. Soc. 127 (1999), no. 5, 1499-1507.

[LMS] A.E. Litvak, V.D. Milman and G. Schechtman, Averages of norms and quasi-norms, Math. Ann. 312 (1998), 95-124.

[LMT] A. E. Litvak, V. D. Milman, N. Tomczak-Jaegermann, When random proportional subspaces are also random quotients, preprint.

[LT] A. E. Litvak, N. Tomczak-Jaegermann, Random aspects of highdimensional convex bodies, GAFA, Lecture Notes in Math., V. 1745, 169190, Springer-Verlag, 2000.

[MT] P. Mankiewicz, N. Tomczak-Jaegermann, Geometry of families of random projections of symmetric convex bodies. Geometric and Functional Analysis, 11 (2001), 1282-1326. 
[M1] V. D. Milman, Almost Euclidean quotient spaces of subspaces of a finite dimensional normed space. Proc. Amer. Math. Soc. 94 (1985), 445-449.

[M2] V. D. Milman, Geometrical inequalities and mixed volumes in the local theory of Banach spaces. Asterisque, 1985, v.131, 373-400.

[M3] V. D. Milman, An inverse form of the Brunn-Minkowski inequality, with applications to the local theory of normed spaces. C. R. Acad. Sci. Paris Sr. I Math. 302 (1986), no. 1, 25-28.

[M4] V. D. Milman, Isomorphic symmetrizations and geometric inequalities. Geometric aspects of functional analysis (1986/87), 107-131, Lecture Notes in Math., 1317, Springer, Berlin, 1988.

[M5] V. D. Milman, Some applications of duality relations. Geometric aspects of functional analysis (1989-90), 13-40, Lecture Notes in Math., 1469, Springer, Berlin, 1991.

[M6] V.D. Milman, Isomorphic Euclidean regularization of quasi-norms in $\mathbb{R}$. C. R. Acad. Sci. Paris, 321 (1996), 879-884.

[MPa1] V. D. Milman, A. Pajor, Entropy methods in asymptotic convex geometry. C. R. Acad. Sci. Paris, Ser. I Math. 329 (1999), no. 4, 303-308.

[MPa2] V. D. Milman, A. Pajor, Entropy and asymptotic geometry of nonsymmetric convex bodies. Adv. Math. 152 (2000), no. 2, 314-335.

[MPi] V. D. Milman, G. Pisier, Banach spaces with a weak cotype 2 property. Israel J. Math. 54 (1986), no. 2, 139-158.

[MS1] V. D. Milman, G. Schechtman, Asymptotic theory of finite-dimensional normed spaces. With an appendix by M. Gromov. Lecture Notes in Mathematics, 1200. Springer-Verlag, Berlin, 1986.

[MS2] V. D. Milman, G. Schechtman, Global versus local asymptotic theories of finite-dimensional normed spaces. Duke Math. J. 90 (1997), no. 1, 73-93.

[PT] A. Pajor, N. Tomczak-Jaegermann, Subspaces of small codimension of finite-dimensional Banach spaces. Proc. Amer. Math. Soc. 97 (1986), no. 4, 637-642.

[Pi] G. Pisier, The Volume of Convex Bodies and Banach Space Geometry. Cambridge University Press, Cambridge 1989. 
[Ro] S. Rolewicz, Metric linear spaces. Monografie Matematyczne, Tom. 56. [Mathematical Monographs, Vol. 56] PWN-Polish Scientific Publishers, Warsaw, 1972.

[Ru] Rudelson M. Distances between non-symmetric convex bodies and the $M M^{*}$-estimate. Positivity, 4 (2000), no. 2, 161-178.

[Sz] S. J. Szarek, Spaces with large distance to $\ell_{\infty}^{n}$ and random matrices. Amer. J. Math. 112 (1990), 899-942.

[T] N. Tomczak-Jaegermann, Banach-Mazur distances and finite-dimensional operator ideals. Pitman Monographs and Surveys in Pure and Applied Mathematics, 38. Longman Scientific \& Technical, Harlow; copublished in the United States with John Wiley \& Sons, Inc., New York, 1989.

Department of Mathematical and Statistical Sciences,

University of Alberta,

Edmonton, Alberta, Canada T6G 2G1,

alexandr@math. ualberta.ca

Department of Mathematics,

Raymond and Beverly Sackler Faculty of Exact Sciences

Tel Aviv University, Tel Aviv, Israel

milman@math.tau.ac.il

Department of Mathematical and Statistical Sciences,

University of Alberta,

Edmonton, Alberta, Canada T6G 2G1,

nicole@ellpspace.math.ualberta.ca 